\title{
A Potentially Useful Galactic Dark Matter Index
}

\author{
Eugene Terry Tatum \\ 760 Campbell Ln. Ste. 106 \#161, Bowling Green, KY 42104, USA \\ Email:ett@twc.com
}

How to cite this paper: Tatum, E.T. (2018) A Potentially Useful Galactic Dark Matter Index. Journal of Modern Physics, 9, 15641567.

https://doi.org/10.4236/jmp.2018.98097

Received: June 25, 2018

Accepted: July 15, 2018

Published: July 18, 2018

Copyright (C) 2018 by author and Scientific Research Publishing Inc. This work is licensed under the Creative Commons Attribution International License (CC BY 4.0).

http://creativecommons.org/licenses/by/4.0/

\begin{abstract}
There have been a number of observational surprises with respect to galactic dark matter-to-visible matter ratios. These surprises confirm our continued lack of understanding of the fundamental nature of dark matter. Because of their apparent close ties with galactic gravitational entropy, at least four recent observations appear to provide the first evidence in support of Verlinde's theory of gravity, dark energy and dark matter as emergent properties. They also appear to correlate with Roger Penrose's gravitational entropy concept, as well as entropy defined in the Flat Space Cosmology (FSC) model. Given the observational support, two different testable versions of a "Dark Matter Index" (DMI) are introduced in this paper, and its utility is discussed in terms of potentially achieving a better understanding of the fundamental nature of dark matter.
\end{abstract}

\section{Keywords}

Dark Matter, Flat Space Cosmology, Cosmology Theory, Gravitational Entropy, Black Holes, DMI

\section{Introduction and Background}

Recent observations have shown a distant exceedingly diffuse galaxy (NGC 1052-DF2) with no discernable dark matter whatsoever [1]. A second report indicates the virial volume of the Milky Way galaxy to have a dark matter-to-visible matter ratio of 23.074-to-1 [2] [3].

Roger Penrose, in his new book [4], introduces the concept of gravitational entropy on pages 256-258. In sharp contrast to entropy of an ideal gas, gravitational and cosmological entropy increases with the ongoing clustering of stars and galaxies. Thus, as Penrose pointed out, black holes (especially supermassive black holes) become huge repositories of total cosmic entropy over the great span of cosmic time. Furthermore, as detailed in "Clues to the Fundamental Nature of Gravity, Dark Energy and Dark Matter" [5], there appears to be a deep 
connection within the Flat Space Cosmology (FSC) model between gravity and cosmic entropy. Thus, FSC appears to be the cosmological model correlate to Verlinde's theory that gravity is an emergent property [6] [7]. The obvious implication, as discussed in the FSC "Clues" paper, is that if gravity is indeed an emergent property, dark energy and dark matter would also likely be emergent properties. If such were the case, dark energy could well be an emergent property of the cosmic system as a whole, and "dark matter" could well be an emergent property of the galactic visible matter. Recently, Verlinde's theory as it may pertain to dark matter, was tested by Brouwer, et al. [8]. Their apparent lensing excess surface density (ESD) analysis of 33,613 galaxies provided the first observational support that Verlinde's theory appears to be correct with respect to galactic dark matter.

Given this observational support for Verlinde's theory, and the apparent deep connections between cosmic entropy and gravity, dark energy and dark matter in FSC, the two additional dark matter observations mentioned in the first paragraph of this paper take on a new significance. This is because they may provide evidence that dark matter observations are closely linked with galactic gravitational entropy. Could it be that the absence of apparent dark matter in the distant and exceedingly diffuse (i.e., gravitationally young) NCG 1052-DF2 galaxy is because it has an exceedingly low gravitational entropy? And could it be that the relative abundance of dark matter in the relatively compact, dense and gravitationally mature Milky Way galaxy (including of course its supermassive black hole), is because it has a high gravitational entropy? If indeed Penrose's concept of gravitational entropy is correct, the answer would seem to be an emphatic "yes!" These additional observations of dark matter in the form of dark matter-to-visible matter ratios of zero and approximately 23-to-1, respectively, would appear to provide the second and third observations in support of Verlinde's theory, and of FSC as an emergent gravity cosmological model. Furthermore, the recent report [9] that dark matter appears to be relatively scarce in the massive star-forming galaxies at high redshifts would appear to be the fourth observation in support of Verlinde's theory and the FSC model presented in the "Clues" paper.

\section{Recommended Dark Matter Index with Discussion}

It is not always easy to quantify the amount of dark matter within a particular galaxy and its dark matter halo. Gravitational lensing, for instance, can be a hit-or-miss proposition, depending upon the alignment of more distant galaxies. However, the above recent observational results apparently in support of Verlinde's theory suggest a possible means of indexing the dark matter of any galaxy for which the following characteristics can be measured: the galactic center intrinsic brightness $I_{B G C}$ and the galactic redshift $S$. This author suggests that these measured characteristics could be expressed in the form of a "Dark Matter Index" (DMI) ratio according to: 


$$
D M I=I_{B G C} / S
$$

If Verlinde, Penrose and the FSC model are indeed correct, one would expect high $D M I$ values to be indicative of high galactic gravitational entropy, high galactic dark matter-to-visible matter ratios, and high excess gravitational lensing powers currently attributed to dark matter. The potential usefulness of such an index could be studied by comparing galaxies in the dark matter data base for which the dark matter-to-visible matter ratio has already been measured, either by gravitational lensing or other means. Even with the more labor-intensive and alignment-dependent gravitational lensing studies of the sort reported by Brouwer, et al, a lensing $D M I$ value (represented as $D M I_{L}$ below) could ultimately prove to have better correlative power than the excess surface density value alone. The proposed relation is

$$
D M I_{L}=E S D_{D M} / S
$$

wherein $E S D_{D M}$ is Brouwer's excess surface density proposed to be attributable to dark matter and $S$ is the galactic redshift. If correlation studies of these $D M I$ and $D M I_{L}$ indices prove them to be accurate quantitative measures of galactic dark matter, this may speed up the process of truly identifying the fundamental nature of dark matter.

\section{Summary and Conclusions}

There have been a number of observational surprises with respect to galactic dark matter-to-visible matter ratios. These surprises confirm our continued lack of understanding of the fundamental nature of dark matter. Because of their apparent close ties with galactic gravitational entropy, at least four recent observations appear to provide the first evidence in support of Verlinde's theory of gravity, dark energy and dark matter as emergent properties. They also appear to correlate with Roger Penrose's gravitational entropy concept, as well as entropy defined in the Flat Space Cosmology (FSC) model. Given the observational support, two different testable versions of a "Dark Matter Index" (DMI) are introduced in this paper, and its utility is discussed in terms of potentially achieving a better understanding of the fundamental nature of dark matter.

\section{Dedications and Acknowledgements}

This paper is dedicated to Dr. Stephen Hawking and Dr. Roger Penrose for their groundbreaking work on black holes and their possible application to cosmology. Dr. Tatum also thanks Dr. Rudolph Schild of the Harvard Center for Astrophysics for his past support and encouragement.

\section{References}

[1] van Dokkum, P., et al. (2018) Nature, 555, 629-632. https://doi.org/10.1038/nature25767

[2] Posti, L. and Helmi, A. (2018) Mass and Shape of the Milky Way's Dark Matter Halo with Globular Clusters from Gaia and Hubble. arXiv:1805.01408v1 [as- 
tro-ph.GA].

[3] Tatum, E.T. (2018) Journal of Modern Physics, 9, 1559-1563.

[4] Penrose, R. (2016) Fashion Faith and Fantasy in the New Physics of the Universe. Princeton University Press, Princeton. https://doi.org/10.1515/9781400880287

[5] Tatum, E.T. and Seshavatharam, U.V.S. (2018) Journal of Modern Physics, 9, 1469-1483. https://doi.org/10.4236/jmp.2018.98091

[6] Verlinde, E. (2010) On the Origin of Gravity and the Laws of Newton. arXiv:1001.0785v1 [hep-th].

[7] Verlinde, E. (2016) Emergent Gravity and the Dark Universe. arXiv:1611.02269v2 [hep-th].

[8] Brouwer, M.M., et al. (2016) First Test of Verlinde's Theory of Emergent Gravity Using Weak Gravitational Lensing Measurements. Monthly Notices of the Royal Astronomical Society, 1-14. arXiv:1612.03034v2 [astro-ph.CO].

[9] Genzel, R., et al. (2017) Nature, 543, 397-401. https://doi.org/10.1038/nature21685 